\title{
Allocution de la présidente entrante, présentée à l'assemblée générale du 10 février 2018
}

De brillantes personnalités du monde de la physique venant de laboratoires académiques se sont succédé à la tête de la Société Française de Physique. Je remercie celles et ceux qui m'ont élue comme première présidente issue du monde de la recherche industrielle. J'en suis très honorée, pour tout dire un peu intimidée et je mesure les attentes liées à ce choix.

Depuis un an, j'ai pu observer le travail et admirer l'engagement constant de Michel Spiro et de toutes les composantes de la SFP. Je tiens à saluer ici leurs nombreuses initiatives et à les remercier pour leur accueil bienveillant.

Sous la présidence de Michel Spiro, de nombreuses actions ont été menées, représentant de réelles avancées par rapport aux ambitions affichées de la SFP depuis plusieurs années. J'en citerai ici quelques-unes qui me paraissent exemplaires et auront vocation à être poursuivies

- L'affichage de valeurs, au cœur de notre activité, favorisant la créativité et l'excellence scientifique associées à l'intégrité, la reconnaissance de la diversité et la science citoyenne.

- La mise en place effective de l'adhésion de laboratoires du monde académique : 50 laboratoires nous ont déjà rejoints à ce jour, l'ambition étant de continuer à en attirer d'autres et de faire adhérer les UFR Sciences des universités.

- La charte de la parité, rédigée par la commission Femmes \& Physique ; cette charte est un pas important dans la reconnaissance de la contribution des femmes au monde scientifique.

- Le lancement de trois nouvelles commissions : "Physique sans frontières », "Physique et Entreprise » et bientôt « Financement et organisation de la recherche ».

- La première édition des Journées RPER (Rencontres nationales Physique Entreprise Recherche) à l'Hôtel de Ville de Paris, réunissant plus de 450 physiciens et physiciennes (de niveau doctorat ou postdoctorat), qui ont pu discuter avec des représentants d'entreprises industrielles, de start-up, d'organismes publics, d'universités et de grandes écoles ; alors que les projections indiquent que moins de la moitié d'entre eux trouvera sa place dans le monde académique, ces rencontres ont permis une meilleure connaissance mutuelle très appréciée des participants.

- Le rapprochement avec la Société Française d'Optique, afin de mener des actions communes et d'amplifier notre visibilité.

- De nombreuses prises de position, souvent conjointes avec d'autres sociétés savantes, comme la toute dernière proposant notre contribution au débat sur les énergies et cosignée avec la Société Chimique de France.

Il est généralement attendu du nouveau président qu'il poursuive la feuille de route de son prédécesseur tout en fixant son propre cap. Lors des séminaires internes de décembre 2016 et 2017, nous avons établi une liste d'actions et de propositions ambitieuses dans une ambiance très chaleureuse et constructive. Je compte tout d'abord m'assurer que nous pourrons mener à bien ces projets, en définissant, en lien avec le bureau et le conseil d'administration, des priorités et un calendrier réaliste. Ces priorités feront l'objet d'un prochain éditorial.

À titre plus personnel, au-delà de l'attention que je porterai naturellement à nos relations avec le monde industriel, j'aimerais contribuer au rajeunissement de la SFP, à son développement dans toutes les régions et à la valorisation de ses actions souvent méconnues.

Pour cela, je souhaite m'appuyer sur toutes les composantes de la SFP et tout particulièrement sur la commission Jeunes. Beaucoup d'idées sont en train de voir le jour au sein de cette commission, comme en témoigne son site (http://jeunes.sfpnet.fr). Les jeunes sont un élément essentiel du renouveau de notre association et une source importante d'énergie ! L'objectif sera de leur donner plus de responsabilités dans l'organisation des événements.

La Société Française de Physique fêtera ses 150 ans en 2023, une occasion de réfléchir ensemble à ce que nous voulons qu'elle soit dans cinq ans et de hiérarchiser nos actions en fonction de cette ambition. Cela demandera l'implication de toutes et tous, car la SFP repose sur notre envie collective d'œuvrer pour le bien de la physique et plus généralement de la science et de ses valeurs, pour les promouvoir au sein de la société.

Je vous remercie par avance pour votre engagement et votre soutien.

Catherine Langlais

Présidente de la Société Française de Physique 